\title{
Direct Detection Image Detector and Electron Counting - A New Tool for High Resolution Imaging of Metal-organic Frameworks
}

\author{
Ming $\operatorname{Pan}^{1}$ \\ ${ }^{1 .}$ Gatan, Inc., Pleasanton, CA, USA
}

Metal-organic frameworks (MOFs) have designable topology, extremely large surface areas and internal cavities that result in many promising applications in gas separation, storage, ion conduction and catalysis, etc. [1-2]. As for any materials, structural characterization is important and essential to fully understand the structure-property-performance relationship.

Compared to diffraction techniques (X-ray or electron), transmission electron microscopy (TEM) can provide not only the periodic crystal structural information but also morphological and non-periodic structures such as size, shape, surface, interface and defects. High resolution TEM imaging can also yield atomic scale structural information. However, it is well-known that MOFs suffer severe structural damage under electron beam irradiation due to their extreme porosity. As a result, image resolution is often determined by the degree of structural damage of the sample instead of electron microscope. To image undamaged structures, or improve the resolution, imaging must be performed under the damage threshold or the critical electron dose on the structures.

Traditional TEM image detectors consist of scintillator, optical fiber/lens and imaging sensor of chargecoupled device (CCD) or complementary metal-oxide semiconductor (CMOS). This type of detectors has limited detective quantum efficiency (DQE), resulting in images with inadequate signal-to-noise ratio (SNR) under low dose conditions.

Direct detection cameras completely eliminate the scintillator and the optical component by exposing an active CMOS sensor directly to the electron beam. This improves the DQE of the cameras. Furthermore by counting individual incoming electrons under low dose condition, additional noises associated with scattering and image readout are eliminated that further boosts the DQE, leading to images with higher SNR even under low dose conditions.

Recent report on atomic resolution TEM imaging of MOF ZIF-8 by using a direct detection camera and electron counting demonstrated two key advantages [3]. First, high SNR for individual image frames can be captured under low dose condition. Second, fast image readout allows correction of sample motion during exposure so that image quality is greatly improved. In figure 1 the left image is the fast Fourier transform (FFT) of the [111] ZIF-8 high resolution image acquired with a tiny electron dose of only 4.1 $\mathrm{e}^{-} / \AA^{2}$, the middle is the measured sample motion during the $3 \mathrm{sec}$ exposure and the right is the FFT of the image after correction of sample motion. The effect of sample motion correction is obvious and critical to restoring the highest image resolution. It is worth pointing out that image readout speed determines the maximum dose rate under which electron counting can be reliably carried out.

Direct detection camera and electron counting also offer an opportunity for quantitative measurement of electron dose accumulated on the TEM samples. This gives insight into the relationship between electron dose and structural damage that is essential to imaging undamaged structures. Direct detection and electron counting have proven to be the new tool for high resolution imaging of MOFs or other extremely 
beam sensitive materials [4].

References:

[1] H. Furukawa et al, Science 341 (2013), 1230444.

[2] J. Cejka in "Metal-Organic Frameworks applications from Catalysis to Gas Storage", ed. D.

Farrusseng, (Wiley-VCH, Weinheim), p. 392.

[3] Y. Zhu et al, Nature Materials published online (2017).

[4] The author wishes to thank Drs. C. Czarnik, J. Ciston, Y. Han, Y. Zhu for many useful discussions.
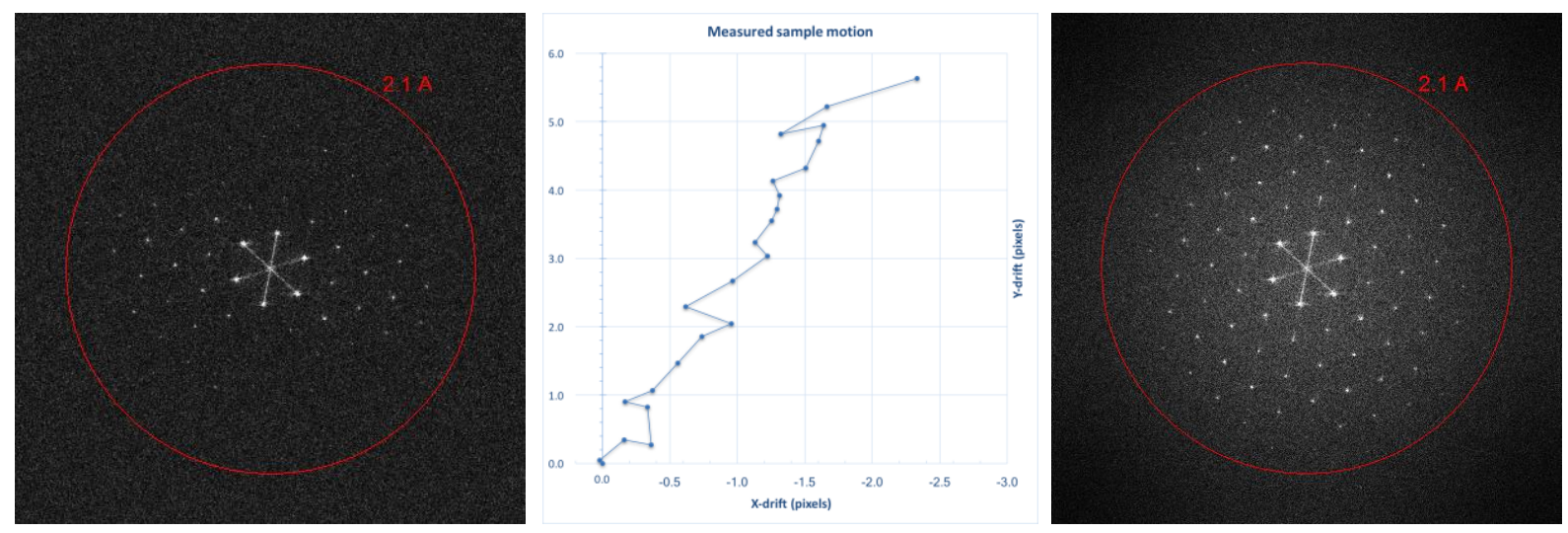

Figure 1. (left) FFT of the [111] ZIF-8 high resolution TEM image as recorded by Gatan K2 ${ }^{\circledR}$ Summit direct detection camera at $300 \mathrm{kV}$. Total electron dose was $\sim 4.1 \mathrm{e}^{-} / \AA^{2}$, exposure $3 \mathrm{~s}$ and TEM magnification $43 \mathrm{kx}$. Information transfer of $2.1 \AA$ was achieved in the horizontal direction. (middle) measured sample motion during the exposure; (right) FFT after sample motion correction showing $2.1 \AA$ information transfer in all directions. 